\title{
Switching on regeneration
}

\author{
Aniek van der Vaart, Sander van den Heuvel \\ Developmental Biology, Department of Biology, Faculty of Science, Utrecht University, Padualaan 8, 3584 CH Utrecht, The Netherlands \\ Correspondence to: Sander van den Heuvel. Developmental Biology, Department of Biology, Faculty of Science, Utrecht University, Padualaan 8,3584 \\ CH Utrecht, The Netherlands. Email: s.j.l.vandenHeuvel@uu.nl.
}

Received: 15 July 2016; Accepted: 10 August 2016; Published: 19 August 2016.

doi: 10.21037/sci.2016.08.05

View this article at: http://dx.doi.org/10.21037/sci.2016.08.05

The limited ability of mammals to repair damaged tissues and organs is well known, and possibilities to improve regeneration are highly desired. Sun et al. (1) (Cell Stem Cell, 2016) recently reported that deleting the Arid1a gene in mice greatly enhances the ability to regenerate the liver and ears after induced injury. Arid1 a forms part of SWI/ SNF chromatin-remodeling complexes, which normally support promoter access for transcription factors that promote differentiation and inhibit proliferation. The findings provide new insight in the control of terminal differentiation and suggest strategies to promote tissue repair.

At some point in our life, the cells of our body still had unlimited potential and were free to choose their future fates. This, however, was quite close to conception; the ability of cells to go all directions is lost early in the development of a complete and complex human being. During development, cells step by step adopt a specific fate, thereby loosing totipotency and gradually becoming completely specialized. The end products are terminally differentiated cells that retain a fixed identity, and permanently shut down their ability to expand through cell proliferation. The permanent cell cycle exit provides a powerful tumor suppressor mechanism, but the downside is a limited ability to repair tissue damage. Some animals, such as urodele amphibians and teleost fish, are famous for their regenerative ability; they can-for instance-regrow a severed limb or tail [reviewed in (2)]. As mammals, we are unfortunately not capable of regenerating body parts; in fact, we are not even that good at repairing organ damage. These abilities may not be as far removed as we think: a remarkable recent study by Sun $e t$ al. shows that deleting a single gene, Arid1a, greatly enhances the ability of mice to regenerate a severely damaged liver or ear wounds (1).
Arid1 a encodes a subunit of a protein complex known as the SWI/SNF (Switch/Sucrose non-fermentable) chromatin-remodeling complex. This complex contains four core subunits, one of which, BRM or BRG (Bramarelated gene), has ATPase activity that is used to move or eject histones, thereby remodeling nucleosomes and regulating DNA access to transcriptional regulators $(3,4)$. SWI/SNF is conserved from yeast to mammals, and with increasing organismal complexity its composition diversified. The ATPase subunit and the position of several proteins associated with the core subunits within the complex can be filled by multiple mutually exclusive proteins in mammals. This diversification is thought to contribute to strict lineage-specific and developmental-stage specific transcriptional regulation (3). Based on subunit composition, BAF (SWI/SNF-A) and PBAF (SWI/SNF-B) complexes are distinguished. Arid1a is a specific factor of the BAF complexes, but the related Arid1b protein can occupy its position in the complex.

Transcription factors and chromatin regulators, including the SWI/SNF complex, act together to enforce terminal differentiation. The Sun et al. paper (1) addresses the question whether SWI/SNF-mediated chromatin remodeling might suppress the regenerative capacity of mammals. They focused on the liver; the only mammalian organ that can substantially regenerate, showing regrowth even when two-thirds is surgically removed. Expression of Arid1A in the liver is shown to be upregulated after birth, while downregulated during regeneration. To examine whether Arid1a reduction enhances regeneration, Sun et al. used a mouse model for liver-specific Arid1a knock out. These mice showed substantially enhanced proliferation and regeneration capacity following surgical liver resection or chemically-induced liver damage. Conversely, 
Arid1a overexpression impaired proliferation and liver regeneration. In addition, inducible whole-body Arid1a deletion is shown to enhance wound healing after ear punching, indicating a more general benefit of Arid1a loss in tissue regeneration (1).

To investigate the underlying molecular mechanisms responsible for improved regeneration, the authors studied SWI/SNF complex composition and promoter binding, as well as gene expression in normal versus knock out liver tissue. This revealed that SWI/SNF complexes remained intact in the absence of Arid1a, with Arid1b taking its place. Chromatin immunoprecipitation sequencing (ChIPseq) showed that Arid1a normally binds at promoters near transcriptional start sites. Arid1a binding motifs and transcriptional targets were found to overlap in particular with those of the C/ebpa, Hnf4a and Foxa2 transcription factors, which maintain liver differentiation and repress proliferation. Loss of Arid1a is shown to alter expression and to reduce chromatin marks of active transcription in a substantial subset of these genes in the liver.

Interestingly, Arid1a immunoprecipitated not only with other SWI/SNF subunits, but also with the transcription factors $\mathrm{E} 2 \mathrm{~F} 4$ and $\mathrm{C} / \mathrm{ebp} \alpha(1)$. E2F4 represses the transcriptional activation of E2F target genes, many of which are associated with the cell division cycle (5). Loss of Arid1a leads to reduced target gene binding of E2F4, acquisition of active chromatin marks and transcriptional upregulation of cell cycle genes. Together, these results support a model in which the Arid1a-SWI/SNF chromatin remodeling complex promotes DNA access of a number of transcription factors that support terminal differentiation and antagonize cell proliferation. The absence of Arid1a leaves the cells not fully differentiated and more susceptible to proliferation.

Though viable and functional, the question remains to what extent the Arid1a knock out tissues remain normal. Arid $1 b$ is likely to substitute substantially for Arid1a without fully replacing it. AT-rich interaction domain (ARID) proteins bind DNA, but not in a sequence-specific manner (6). They likely interact with transcription factors, nucleosomes, and other chromatin regulators to recruit SWI/SNF complexes to specific promoters. Genomewide mapping of Arid1a, Arid1b and Arid2 DNA binding in the human liver cancer cell line HepG2 showed a high degree of overlap in binding sites, which could explain redundancies and cause competition between the proteins (7). However, unique sites for each of the Arid proteins were also detected, indicating that distinct SWI/SNF complexes can regulate transcription gene-specifically. While not addressed in the current study, the subset of differentiation and cell cycle related genes that show the greatest changes upon Arid1a loss may not be able to recruit Arid1b-SWI/SNF.

It will be interesting to find out how broad the enhanced regeneration effect of Arid1a loss really is. Instead of hepatocytes, could completely post-mitotic cardiomyocytes or neurons be persuaded to proliferate? This will most likely require multiple gene alterations. Interestingly, transient inactivation of the Retinoblastoma $(R b)$ and $A R F$ tumor suppressor genes was previously shown to induce dedifferentiation, cell cycle re-entry, and subsequent redifferentiation of post-mitotic muscle cells (8). The efficiency of this regeneration however is low; $29 \%$ of the myocytes dedifferentiated and re-entered the cell cycle and some, but not all, of these cells redifferentiated after re-expression of $\mathrm{pRb}$. Combined transient loss of $R b, A R F$ and SWI/SNF-Arid1a might increase the regeneration capability. Indeed, the SWI/SNF complex, $R b$ and other cell cycle regulators were shown to cooperate in cell cycle exit and differentiation control in C. elegans. The combined loss of $C$. elegans $\mathrm{Rb}, \mathrm{SCF}^{\beta-\mathrm{TrCP}}$ and SWI/SNF subunits leads to severe overproliferation of muscle precursor cells, while interfering with the single genes has limited effects (9). These and other data point to substantially overlapping levels of control over cell proliferation and differentiation.

Possibly tempering therapeutic hope is the important tumor suppressor function of the SWI/SNF complex, which likely resides in its important role in lineage specification and terminal differentiation. Close to $20 \%$ of human cancers were found to contain loss of function mutations in at least one of the SWI/SNF subunit genes (10). The mutation frequencies differ greatly between cancer types and SWI/ SNF subunits, emphasizing the importance of cellular context and SWI/SNF complex composition. Arid1a is the most frequently mutated SWI/SNF subunit; mutations are most often found in ovarian clear cell carcinoma and endometrial carcinoma, but are also frequently present in several other cancer types (10). Notably, ubiquitous or liver-specific Arid1a knock out mice did not show tumor formation, not even after 1 year. This does not negate a tumor suppression function of Arid1a; multiple other gene deregulations are likely needed to induce cancer formation. Therapeutic strategies targeting SWI/SNF function in tissue regeneration or cancer treatment will require a deep understanding of the recruitment and subunit variability of the SWI/SNF complex. The paper by Sun et al. has given new insight into the importance of epigenetic regulation by 
the SWI/SNF complex in tissue regeneration and highlights new possibilities for future research directions.

\section{Acknowledgements}

We acknowledge financial support from Worldwide Cancer Research, grant 14-1294 to S van den Heuvel, and funding from the Netherlands Organization of Scientific Research (NWO).

\section{Footnote}

Conflicts of Interest: The authors have no conflicts of interest to declare.

Comment on: Sun X, Chuang JC, Kanchwala M, et al. Suppression of the SWI/SNF component Arid1a promotes mammalian regeneration. Cell Stem Cell 2016;18:456-66.

\section{References}

1. Sun X, Chuang JC, Kanchwala M, et al. Suppression of the SWI/SNF component Arid1a promotes mammalian regeneration. Cell Stem Cell 2016;18:456-66.

2. Tornini VA, Poss KD. Keeping at arm's length during

doi: $10.21037 /$ sci.2016.08.05

Cite this article as: van der Vaart A, van den Heuvel S. Switching on regeneration. Stem Cell Investig 2016;3:41. regeneration. Dev Cell 2014;29:139-45.

3. Ho L, Crabtree GR. Chromatin remodelling during development. Nature 2010;463:474-84.

4. Roberts CW, Orkin SH. The SWI/SNF complex-chromatin and cancer. Nat Rev Cancer 2004;4:133-42.

5. Dyson NJ. RB1: a prototype tumor suppressor and an enigma. Genes Dev 2016;30:1492-502.

6. Patsialou A, Wilsker D, Moran E. DNA-binding properties of ARID family proteins. Nucleic Acids Res 2005;33:66-80.

7. Raab JR, Resnick S, Magnuson T. Genome-wide transcriptional regulation mediated by biochemically distinct SWI/SNF complexes. PLoS Genet 2015;11:e1005748.

8. Pajcini KV, Corbel SY, Sage J, et al. Transient inactivation of $\mathrm{Rb}$ and $\mathrm{ARF}$ yields regenerative cells from postmitotic mammalian muscle. Cell Stem Cell 2010;7:198-213.

9. Ruijtenberg S, van den Heuvel S. G1/S inhibitors and the SWI/SNF complex control cell-cycle exit during muscle differentiation. Cell 2015;162:300-13.

10. Kadoch C, Hargreaves DC, Hodges C, et al. Proteomic and bioinformatic analysis of mammalian SWI/SNF complexes identifies extensive roles in human malignancy. Nat Genet 2013;45:592-601. 